\title{
Underscreened Kondo lattice model applied to heavy fermion uranium compounds
}

\author{
N. B. Perkins, ${ }^{1,2}$ M. D. Núñez-Regueiro, ${ }^{3, *}$ B. Coqblin, ${ }^{3}$ and J. R. Iglesias ${ }^{4}$ \\ ${ }^{1}$ Institute fur Theoretische Physik, TU Braunschweig, Mendelssohnstrasse 3, 38106 Braunschweig, Germany \\ ${ }^{2}$ Bogoliubov Laboratory of Theoretical Physics, JINR, 141980 Dubna, Russia \\ ${ }^{3}$ Laboratoire de Physique des Solides, Université Paris-Sud, UMR-8502 CNRS, 91405 Orsay, France \\ ${ }^{4}$ Instituto de Física, Universidade Federal do Rio Grande do Sul, 91501-970 Porto Alegre, Brazil
}

(Received 13 April 2007; revised manuscript received 12 June 2007; published 5 September 2007)

\begin{abstract}
We present theoretical results for the underscreened Kondo lattice model with localized $S=1$ spins coupled to a conduction band through a Kondo coupling, $J_{K}$, and interacting among them ferromagnetically. We use a fermionic representation for the spin operators and expand the Hamiltonian in terms of bosonic fields. For large values of $J_{K}$, we obtain a ferromagnetically ordered solution and a Kondo regime with a Kondo temperature, $T_{K}$, larger than the Curie temperature, $T_{C}$. This finding suggests a scenario for a coexistence of Kondo effect and ferromagnetic order. In some uranium compounds, such as $\mathrm{UTe}$ or $\mathrm{UCu}_{0.9} \mathrm{Sb}_{2}$, this kind of coexistence has been experimentally observed: they order ferromagnetically with a Curie temperature of order $T_{C} \sim 100 \mathrm{~K}$ and exhibit a Kondo behavior for $T>T_{C}$. The proposed underscreened Kondo lattice model accounts well for the coexistence between magnetic order and Kondo behavior and yields to a new "ferromagnetic Doniach diagram."
\end{abstract}

DOI: 10.1103/PhysRevB.76.125101

PACS number(s): 71.27.+a, 75.30.Mb, 75.20.Hr, 75.10.-b

\section{INTRODUCTION}

The Kondo lattice (KL) model is one of the fundamental microscopic models for studying the properties of strongly correlated electron systems, and a large amount of theoretical work was carried out on this problem in recent years (for a review, see Ref. 1). This model is widely used to describe the physics of intermetallic heavy fermion compounds based either on rare earth elements or on actinides. ${ }^{2}$ In heavy fermion materials there are two different types of electrons: conduction electrons from outer atomic orbitals, and strongly correlated electrons from inner $f$ orbitals, the latter ones being generally localized. The KL model describes the interaction between these two electronic subsystems in the limit when $f$ electrons are completely localized and form a lattice of localized spins.

Historically, KL model has been proposed to account for properties of cerium compounds, where a competition between Kondo effect and magnetic order has been experimentally observed. Such competition gives rise to a rich phase diagram with various quantum phase transitions. KL model has been proven to be an appropriate tool for describing these quantum transitions at different values of external parameters such as band filling, pressure, magnetic field, or temperature. 3,4

In most cerium compounds, $\mathrm{Ce}$ ions are in the localized $4 f^{1}$ configuration corresponding to spin $S=1 / 2$. This localized spin couples antiferromagnetically, via an on-site exchange interaction, $J_{K}$, to the conduction electron spin density. At very low temperatures the localized spin $S=1 / 2$ is completely screened by the conduction electrons, leading to the formation of coherent Kondo spin-singlet state. Besides, the local coupling between $f$ spins and conduction electrons may give rise to a magnetic order through the RudermanKittel-Kasuya-Yosida (RKKY) interaction. This interaction is usually added to the $\mathrm{KL}$ model as an additional intersite interaction between $f$ spins. ${ }^{5,6}$
The competition between the magnetic order and the Kondo effect was first considered by Doniach. ${ }^{3,7} \mathrm{He}$ proposed a phase diagram with a quantum phase transition between a magnetically ordered phase and a nonmagnetic Kondo phase. Doniach phase diagram was later extended ${ }^{3}$ to include the short-range magnetic correlations that survive inside the Kondo phase. ${ }^{8}$ From an experimental point of view, the competition between magnetic order and Kondo effect has been observed in many cerium and ytterbium compounds, which yields a set of very rich phase diagrams with various quantum phase transitions under pressure (see, for example, Ref. 4 and references therein).

In this paper, we focus on the physical properties of uranium compounds. This is another class of heavy fermion systems, which show very rich behavior, quite different from cerium compounds. It is peculiar of uranium compounds that they exhibit numerous coexistence phenomena, the most prominent of which is the coexistence of magnetic order with Kondo effect ${ }^{9-13}$ or the coexistence of the magnetic order with superconductivity. ${ }^{14,15}$ We will be primarily interested in the coexistence between ferromagnetic order and Kondo behavior, as, to our knowledge, this effect has been somewhat overlooked from a theoretical point of view.

Let us briefly describe the experimental situation. The first experimental evidence of the coexistence between Kondo behavior and ferromagnetic order in the dense Kondo compound UTe has been obtained long time ago. ${ }^{9}$ More recently, this coexistence has been observed in $\mathrm{UCu}_{0.9} \mathrm{Sb}_{2}{ }^{11}$ and $\mathrm{UCo}_{0.5} \mathrm{Sb}_{2} .{ }^{12,13}$ All these systems undergo a ferromagnetic ordering at the relatively high Curie temperatures of $T_{C}$ $=102 \mathrm{~K}(\mathrm{UTe}), T_{C}=113 \mathrm{~K}\left(\mathrm{UCu}_{0.9} \mathrm{Sb}_{2}\right)$, and $T_{C}=64.5 \mathrm{~K}$ $\left(\mathrm{UCo}_{0.5} \mathrm{Sb}_{2}\right)$. Above the ordering temperatures, i.e., in the expected paramagnetic region, these materials exhibit a Kondo-like logarithmic decrease of the electrical resistivity, indicating a Kondo behavior. This logarithmic variation extends down to the ferromagnetic Curie temperature, $T_{C}$, suggesting that the Kondo behavior survives inside the ferro- 
magnetic phase, implying that the ferromagnetic order and the Kondo behavior do coexist. This coexistence, together with the large Curie temperatures, are clearly unusual features that cannot be explained by the standard KL model. ${ }^{18}$

Therefore, as a minimal model to describe the Kondoferromagnetism coexistence, we propose an underscreened Kondo lattice model which, we argue, is appropriate to describe the $5 f^{2}$ configuration of uranium ions.

The underscreened Kondo lattice (UKL) model consists of a periodic lattice of magnetic atoms with $S=1$ interacting with a spin density of conduction electrons via an on-site antiferromagnetic Kondo coupling. In addition, the localized spins at neighboring sites interact ferromagnetically with each other. In this case the Kondo effect does not lead to a complete screening of the localized spins, and the ferromagnetic exchange between the (underscreened) spins may indeed lead to the formation of ferromagnetic order.

We warn that the choice of the model for the electronic structure of uranium compounds is a question not settled yet. Magnetism in these compounds undoubtedly comes from $5 f$ electrons - this has been proven by many experimental observations, e.g., by form-factor studies in neutron scattering. At the same time, $5 f$ electron states in uranium compounds are in a crossover region between localized and itinerant behavior, and the degree of localization depends strongly on a subtle balance between the electronic structure, the effect of correlations, and crystal field effects. It is often difficult to decide, on the basis of the experimental data, between a local Kondo behavior corresponding to a $5 f^{n}$ configuration and a mixed-valence situation. One example is provided by uranium monochalcogenides: US lies closest to the itinerant side for the $5 f$ electrons, USe is in the middle, and UTe is the closest to the localized side. ${ }^{19-21}$ Recent photoemission experiments on UTe have been interpreted as favoring itinerant magnetism, ${ }^{22}$ but the magnetic moments deduced from magnetic susceptibility experiments in this compound are close to the free ion values of uranium, which implies that the $5 f$ electrons are relatively well localized in UTe. ${ }^{10,19}$ Moreover, the dual nature of the $5 f$ electrons, assuming two localized $5 f$ electrons and one delocalized one, has been considered by Zwicknagl et al. ${ }^{23,24}$ who have obtained by band calculations a mass enhancement factor in good agreement with experiment in $\mathrm{UPt}_{3}$ and $\mathrm{UPd}_{2} \mathrm{Al}_{3}$ and by Schoenes et al. ${ }^{19,20}$ who have carefully analyzed the variation of the localization of the $5 f$ electrons with concentration and pressure in diluted US and UTe. The electronic structure of uranium and plutonium monochalcogenides has been also studied by dynamical mean field theory (DMFT) calculations. ${ }^{25}$ So, the appropriate description of the electronic structure for uranium compounds is a challenging problem and depends strongly on the considered system. Here we restraint ourselves to the study of the UKL model applied to uranium compounds such as UTe, when the uranium ions are relatively well localized and can be correctly described within a $5 f^{2}$ configuration, in which the two $5 f$ electrons are bound into spin $S=1$.

Besides its applicability to the physics of ferromagnetic uranium compounds, UKL model is also interesting on its own. It is one of the theoretical models which can capture the physics of the lattice of underscreened magnetic moments in a metal, yet it attracted much less attention than the underscreened Kondo impurity model, for which there there exist various theoretical studies, ${ }^{26}$ and an exact solution has been obtained by the Bethe ansatz. ${ }^{27,28}$ There has been only few studies of the UKL. The UKL model in the form which we use here was first proposed in Ref. 29. Magnetism and superconductivity in one dimensional UKL model has been studied in Ref. 30. The pseudogap formation in the UKL model has been studied in a large- $N$ limit in Ref. 31 .

The crucial step in our analysis of the UKL model lies in the choice of the fermionic representation of localized spins $S=1$. We model them by two degenerate $f$ orbitals with one $f$ electron each. At each site these two electrons are bound into $S=1$ due to the strong on-site Hund's coupling. Our fermionic representation projects out singlet states and satisfies spin algebra in the triplet Hilbert subspace. ${ }^{32}$ In other words, all possible spin transitions that leave the system in the triplet subspace, $\left|S=1, S_{z}\right\rangle$ are equivalently described in terms of auxiliary fermionic operators.

We show that the UKL model exhibits two continuous transitions (more precisely, sharp crossovers): the first one, at $T=T_{K}$, to a nonmagnetic Kondo state with a pseudogap in the $f$-electron density of states, and the second one, at $T=T_{C}$, to a ferromagnetic state. We find that at strong Kondo coupling ferromagnetism and Kondo effect do coexist. We evaluate the Kondo screening, magnetic moments of localized spins, and conduction electrons for various band fillings in a wide range of coupling parameters and obtain the phase diagram with the regions of Kondo-ferromagnetic coexistence, nonmagnetic Kondo behavior, and pure ferromagnetism. This phase diagram can be considered as a ferromagnetic "Doniach" diagram for the UKL model.

The paper is organized as follows: In Sec. II, we present the model, introduce the fermionic representation for spin operators, derive the Green's functions, and perform a selfconsistent analysis. The results of the calculations at zero and finite temperatures are discussed in Sec. III. In Sec. III E, we present the ferromagnetic "Doniach diagram" for our model. The conclusion contains the discussion of the main results and the comparison with the experimental data for the uranium compounds.

\section{UNDERSCREENED KONDO LATTICE MODEL}

\section{A. Fermionic representation of localized spins}

We first introduce and discuss in detail the fermionic representation for localized spins $S=1$, made out of two fermions on degenerate $f$ orbitals. The two fermions couple into $S=1$ due to the strong on-site Hund's interaction. We introduce a fermionic representation in the constrained Hilbert space which contains only triplet spin states, dropping out all spin-singlet states.

The projection of the spin-singlet states is justified due to the correlation nature of $f$ electrons. The strong Hund's coupling favors the triplet states with energy $E_{t}$ with respect to the singlet states with energy $E_{s}$, and to the states with two electrons on a single orbital, with even higher energy $E_{d}$.

Then, considering just states with $S=1$ the transformation between different $S_{z}\left(\left|1, S_{z}\right\rangle\right)$ projections is unambiguously described in terms of fermionic operators as ${ }^{32}$ 


$$
\begin{gathered}
\frac{1}{2}\left(f_{1 \uparrow}^{\dagger} f_{1 \uparrow} f_{2 \downarrow}^{\dagger} f_{2 \downarrow}+f_{1 \downarrow}^{\dagger} f_{1 \downarrow} f_{2 \uparrow}^{\dagger} f_{2 \uparrow}+f_{1 \uparrow}^{\dagger} f_{1 \downarrow} f_{2 \downarrow}^{\dagger} f_{2 \uparrow}\right. \\
\left.+f_{1 \downarrow}^{\dagger} f_{1 \uparrow} f_{2 \uparrow}^{\dagger} f_{2 \downarrow}\right):|1,0\rangle \rightarrow|1,0\rangle, \\
f_{1 \uparrow}^{\dagger} f_{1 \uparrow} f_{2 \uparrow}^{\dagger} f_{2 \uparrow}:|1,1\rangle \rightarrow|1,1\rangle, \\
f_{1 \downarrow}^{\dagger} f_{1 \downarrow} f_{2 \downarrow}^{\dagger} f_{2 \downarrow}:|1,-1\rangle \rightarrow|1,-1\rangle, \\
\frac{1}{2}\left(f_{1 \uparrow}^{\dagger} f_{1 \uparrow} f_{2 \downarrow}^{\dagger} f_{2 \uparrow}+f_{1 \downarrow}^{\dagger} f_{1 \uparrow} f_{2 \uparrow}^{\dagger} f_{2 \uparrow}\right):|1,1\rangle \rightarrow|1,0\rangle, \\
\frac{1}{2}\left(f_{1 \downarrow}^{\dagger} f_{1 \downarrow} f_{2 \downarrow}^{\dagger} f_{2 \uparrow}+f_{1 \downarrow}^{\dagger} f_{1 \uparrow} f_{2 \downarrow}^{\dagger} f_{2 \downarrow}\right):|1,0\rangle \rightarrow|1,-1\rangle, \\
\frac{1}{2}\left(f_{1 \downarrow}^{\dagger} f_{1 \downarrow} f_{2 \uparrow}^{\dagger} f_{2 \downarrow}+f_{1 \uparrow}^{\dagger} f_{1 \downarrow} f_{2 \downarrow}^{\dagger} f_{2 \downarrow}\right):|1,-1\rangle \rightarrow|1,0\rangle, \\
\frac{1}{2}\left(f_{1 \uparrow}^{\dagger} f_{1 \uparrow} f_{2 \uparrow}^{\dagger} f_{2 \downarrow}+f_{1 \uparrow}^{\dagger} f_{1 \downarrow} f_{2 \uparrow}^{\dagger} f_{2 \uparrow}\right):|1,0\rangle \rightarrow|1,1\rangle .
\end{gathered}
$$

where $f_{\alpha \sigma}^{\dagger}$ and $f_{\alpha \sigma}$ are creation and annihilation operators for $f$ electrons, carrying spin and orbital indices, $\sigma$ and $\alpha(\alpha$ $=1,2)$, respectively. In terms of spin operators, the transitions represented by Eqs. (1) can be expressed as

$$
\begin{gathered}
1-S_{z}^{2}:|1,0\rangle \rightarrow|1,0\rangle, \\
\frac{1}{2} S_{z}\left(S_{z}-1\right):|1,-1\rangle \rightarrow|1,-1\rangle, \\
\frac{1}{2} S_{z}\left(S_{z}+1\right):|1,1\rangle \rightarrow|1,1\rangle, \\
-\frac{1}{2} S_{z} S^{-}:|1,0\rangle \rightarrow|1,-1\rangle, \\
-\frac{1}{2} S^{+} S_{z}:|1,-1\rangle \rightarrow|1,0\rangle, \\
\frac{1}{2} S^{-} S_{z}:|1,1\rangle \rightarrow|1,0\rangle, \\
\frac{1}{2} S_{z} S^{+}:|1,0\rangle \rightarrow|1,1\rangle .
\end{gathered}
$$

Note that there is no transition $|1,1\rangle \leftrightarrow|1,-1\rangle$. The equivalence between fermionic and spin representations should be interpreted as the equivalence of matrix elements between corresponding states.

\section{B. Model}

Our model Hamiltonian is the following:

$H=\sum_{\mathbf{k} \sigma}\left(\epsilon_{\mathbf{k}}-\mu\right) c_{\mathbf{k} \sigma}^{\dagger} c_{\mathbf{k} \sigma}+\sum_{i \sigma \alpha} E_{0} n_{i \sigma}^{f_{\alpha}}+J_{K} \sum_{i} \mathbf{S}_{i} \sigma_{i}+\frac{1}{2} J_{H} \sum_{i j} \mathbf{S}_{i} \mathbf{S}_{j}$.

The first term represents the conduction band with dispersion energy $\epsilon_{k}$. We assume that the band has a width $2 D$ and the density of states for conduction electrons is $1 / 2 D$ in the interval $[-D, D]$ and zero otherwise. The operators $c_{\mathbf{k} \sigma}^{\dagger}\left(c_{\mathbf{k} \sigma}\right)$ correspond to delocalized Bloch states with spin $\sigma$, while $\mu$ is the bare electron chemical potential. The second term describes the energy of localized levels and $E_{0}$ can be considered as a fictitious chemical potential, i.e., a Lagrange multiplier for auxiliary $f$ fermions. The actual value of $E_{0}$ is fixed by a local constraint $n_{f}=\sum_{i \sigma \alpha} n_{i \sigma}^{f_{\alpha}}=2$ for the number of $f$ electrons per site. The third term is the antiferromagnetic on-site Kondo coupling, with $J_{K}>0$, between localized $f$ spins, $S_{i}=1$, and conduction electrons $\sigma_{i}=1 / 2$ spins. The spin operators $\sigma_{i}$ can be written in terms of the fermionic operators in a standard way: $\sigma_{i}^{+}=c_{i \uparrow}^{+} c_{i \downarrow}, \quad \sigma_{i}^{-}=c_{i \downarrow}^{+} c_{i \uparrow}, \quad \sigma_{i}^{z}$ $=\frac{1}{2}\left(n_{i \uparrow}^{c}-n_{i \downarrow}^{c}\right)$. The last term in Eq. (3) is a ferromagnetic intersite interaction, $J_{H}<0$, between localized $f$ magnetic moments. ${ }^{34}$

Using the fermionic representation for both $f$ and conduction electron spin, we can rewrite the Hamiltonian (3) in terms of $f$ and $c$ electronic operators. As the expression of the Hamiltonian in terms of fermionic operators is rather lengthy, we show here in detail just the most relevant term, the transverse part of the Kondo coupling $\frac{1}{2} J_{K}\left(\sigma_{i}^{+} S_{i}^{-}+\sigma_{i}^{-} S_{i}^{+}\right)$, which reads, when expressed in the fermionic representation,

$$
\begin{aligned}
\frac{1}{2} J_{K}\left(\sigma_{i}^{+} S_{i}^{-}+\sigma_{i}^{-} S_{i}^{+}\right)= & \frac{1}{2} J_{K}\left[c _ { i \uparrow } ^ { + } c _ { i \downarrow } \left(f_{i 1 \uparrow}^{+} f_{i 1 \uparrow} f_{i 2 \downarrow}^{+} f_{i 2 \uparrow}+f_{i 1 \downarrow}^{+} f_{i 1 \uparrow} f_{i 2 \uparrow}^{+} f_{i 2 \uparrow}\right.\right. \\
& \left.+f_{i 1 \uparrow}^{+} f_{i 1 \downarrow} f_{i 2 \downarrow}^{+} f_{i 2 \uparrow}+f_{i 1 \uparrow}^{+} f_{i 1 \uparrow} f_{i 2 \downarrow}^{+} f_{i 2 \downarrow}\right) \\
& +c_{i \downarrow}^{+} c_{i \uparrow}\left(f_{i 1}^{+} f_{i 1 \downarrow} f_{i 2 \uparrow}^{+} f_{i 2 \downarrow}+f_{i 1 \uparrow}^{+} f_{i 1 \downarrow} f_{i 2 \downarrow}^{+} f_{i 2 \downarrow}\right. \\
& \left.\left.+f_{i 1 \uparrow}^{+} f_{i 1 \uparrow} f_{i 2 \uparrow}^{+} f_{i 2 \downarrow}+f_{i 1 \uparrow}^{+} f_{i 1 \downarrow} f_{i 2 \uparrow}^{+} f_{i 2 \uparrow}\right)\right] .
\end{aligned}
$$

Now we define the relevant bosonic fields to describe the Kondo effect: we introduce the operator $\hat{\lambda}_{i \sigma}=\Sigma_{\alpha} \hat{\Lambda}_{i \sigma}^{\alpha}$ $=\Sigma_{\alpha} c_{i \sigma}^{+} f_{i \sigma}^{\alpha}$ which couples electrons and auxiliary fermions at the same site. Using this definition, we rewrite Eq. (4) as

$$
\begin{aligned}
\frac{1}{2} J_{K}\left(\sigma_{i}^{+} S_{i}^{-}+\sigma_{i}^{-} S_{i}^{+}\right)= & \frac{1}{2} J_{K}\left(-\lambda_{i \uparrow}^{2} \lambda_{i \downarrow}^{* 2} n_{1}-\lambda_{i \uparrow}^{1} \lambda_{i \downarrow}^{* 1} n_{2}-\lambda_{i \downarrow}^{2} \lambda_{i \uparrow}^{* 2} n_{1}\right. \\
& \left.-\lambda_{i \downarrow}^{1} \lambda_{i \uparrow}^{* 1} n_{2}\right)=-\frac{1}{2} J_{K} \sum \sum_{\alpha \sigma} \lambda_{i \sigma}^{\alpha} \lambda_{i \bar{\sigma}}^{* \alpha} n_{\bar{\alpha}} .
\end{aligned}
$$

Moreover, in order to describe the magnetic properties of the system, we introduce the operators of magnetization for both $f$ and $c$ subsystems: $M_{i}=S_{i}^{z}=\frac{1}{2}\left(n_{i \uparrow}^{f}-n_{i \dagger}^{f}\right)$ and $m_{i}=\sigma_{i}^{z}$ $=\frac{1}{2}\left(n_{i \uparrow}^{c}-n_{i \downarrow}^{c}\right)$, respectively.

We restrict our consideration to self-consistent analysis (equivalent to slave boson mean-field description), and evaluate all physical quantities in terms of these bosonic fields. We then introduce four real order parameters $\lambda_{\sigma}$ $=\left\langle\hat{\lambda}_{i \sigma}\right\rangle, M=\left\langle M_{i}\right\rangle$, and $m=\left\langle m_{i}\right\rangle$, where $\langle\cdots\rangle$ denotes the thermal average. The nonzero values of $\langle M\rangle$ and $\langle m\rangle$ describe the magnetic phase with nonzero total magnetization, while a nonzero $\lambda_{\sigma}$ describes the Kondo effect and the formation of the heavy fermion state. The limits of this approach are discussed at the end of this subsection.

Within this mean field (MF) approximation, and using the fact that the total number of $f$ electrons in each sublevel is $n_{\alpha}=1$, the Hamiltonian (3) is expressed in terms of the four order parameters $\left\langle\lambda_{\sigma}\right\rangle,\langle M\rangle$, and $\langle m\rangle$ as

$$
\begin{aligned}
H= & \sum_{\mathbf{k} \sigma} \varepsilon_{\mathbf{k} \sigma} c_{\mathbf{k} \sigma}^{+} c_{\mathbf{k} \sigma}+\sum_{i \alpha \sigma} E_{0 \sigma} n_{i \alpha \sigma}-\frac{1}{2} J_{K} \sum_{i \alpha \sigma}\left(\left\langle\lambda_{\bar{\sigma}}\right\rangle \lambda_{i \sigma}^{\alpha}+\text { H.c. }\right) \\
& +2 J_{K} N \sum_{\sigma}\left\langle\lambda_{\bar{\sigma}}\right\rangle\left\langle\lambda_{\sigma}\right\rangle-J_{K} N\langle m\rangle\langle M\rangle-\frac{1}{2} J_{H} N z\langle M\rangle^{2},
\end{aligned}
$$

where

$$
\varepsilon_{\mathbf{k} \sigma}=\varepsilon_{\mathbf{k}}+\Delta_{\sigma}, \quad E_{0 \sigma}=E_{0}+\Lambda_{\sigma},
$$




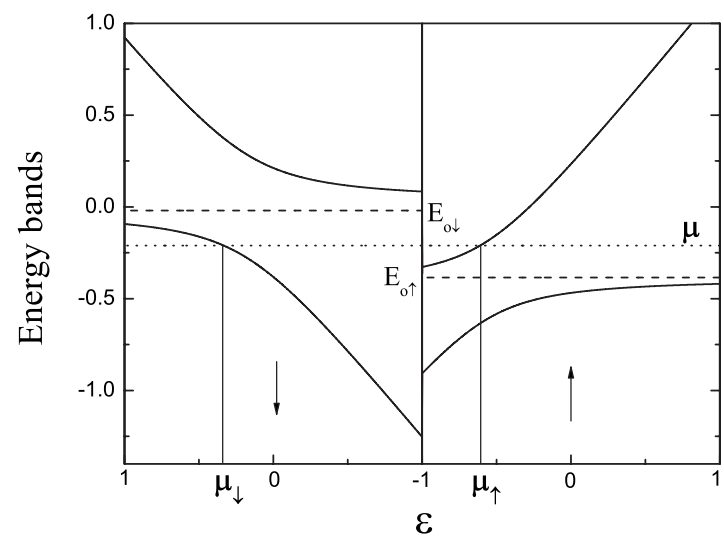

FIG. 1. Schematic plot of the band structure: for each direction of spin there are two hybridized $E_{+}^{\sigma}$ and $E_{-}^{\sigma}(k)$ bands (thick solid lines) and one nonhybridized $f$ level $E_{0 \sigma}$ (dashed line). The left panel corresponds to down spins and the right one to up spins. The thin dotted line corresponds to the Fermi level.

$$
\begin{gathered}
\Delta_{\sigma}=J_{K} \sigma\langle M\rangle, \quad \sigma= \pm 1 / 2, \\
\Lambda_{\sigma}=J_{K} \sigma\langle m\rangle-\frac{1}{2} J_{K}\left\langle\lambda_{\sigma}\right\rangle\left\langle\lambda_{\bar{\sigma}}\right\rangle+J_{H} z \sigma\langle M\rangle,
\end{gathered}
$$

$z$ being the number of nearest neighbors. In the presence of nonzero magnetization the bands for up and down spins in both $f$ and $c$ subsystems are shifted from each other: parameters $\Delta_{\sigma}$ and $\Lambda_{\sigma}$ define the energy shift for itinerant bands and localized $f$ levels, respectively. The shifts for $f$ and $c$ bands are in opposite directions because of the antiferromagnetic Kondo coupling between them.

For each direction of spin, the diagonalization of the Hamiltonian (6) yields one nonhybridized $f$ state with energy $E_{0 \sigma}$ and two hybridized bands with the quasiparticle energy dispersion given by

$$
E_{ \pm}^{\sigma}(\mathbf{k})=\frac{1}{2}\left[E_{0 \sigma}+\varepsilon_{\mathbf{k} \sigma} \pm \sqrt{\left(E_{0 \sigma}-\varepsilon_{\mathbf{k} \sigma}\right)^{2}+8 \alpha_{\bar{\sigma}}^{2}}\right]
$$

where $\alpha_{\bar{\sigma}}=-\frac{1}{2} J_{K}\left\langle\lambda_{\bar{\sigma}}\right\rangle$. The \pm refers to the upper and lower hybridized bands. The hybridization gap in the energy spectrum $\Gamma_{\sigma}=2 \sqrt{2} \alpha_{\bar{\sigma}}=\sqrt{2} J_{K} \lambda_{\bar{\sigma}}$ is spin dependent and is only present as long as $\lambda_{\sigma}$ is nonzero. The schematic plot of the band structure is presented in Fig. 1.

The energy spectra $E_{ \pm}^{\sigma}(\mathbf{k})$ depend on a set of external parameters such as the Kondo coupling, $J_{K}$, and the exchange interaction, $J_{H}$, the conduction-band filling, $n_{c}$, the temperature $T$, and a set of internal parameters, $\mu$ and $E_{0}$, which should be calculated self-consistently.

The present approach is equivalent to other MF theories developed for the $S=1 / 2 \mathrm{KL}$ model, such as saddle-point approximation in the path-integral calculation, performed by Coleman and Andrei, ${ }^{35}$ or large- $N$, slave boson saddle-point approximation by Burdin et al. ${ }^{35}$ All these approximate methods require some caution. Particularly, MF approximation neglects magnetic fluctuations, and the system may possess spurious charge fluctuations such that the exact constraint $f_{\uparrow}^{\dagger} f_{\uparrow}+f_{\downarrow}^{\dagger} f_{\downarrow}=2$ is actually satisfied only on average.
The validity of MF and slave boson approaches for $\mathrm{KL}$ model with localized spin $S=1 / 2$ has been extensively discussed in Refs. 5 and 36. It has been shown that the MF procedure becomes exact when the original model is extended to $N \rightarrow \infty$ different flavors of localized electrons. Still, it was found in the same paper that the MF procedure captures the correct low-temperature physics even for the actual case of $N=2(S=1 / 2)$. By the same reasons, the MF approximation should work in our case where $N=3(S=1)$.

Below we will study the model (6) by employing equations of motion for the Green's function for localized and itinerant spins. All propagators will be written in the fermionic representation.

\section{Green's functions}

We introduce the following notation for the retarded Green's functions: $F_{\alpha \alpha}^{\sigma}=\left\langle\left\langle f_{\alpha \sigma} ; f_{\alpha \sigma}^{\dagger}\right\rangle\right\rangle$ and $F_{\alpha \beta}^{\sigma}=\left\langle\left\langle f_{\alpha \sigma} ; f_{\beta \sigma}^{\dagger}\right\rangle\right\rangle$ for localized $f$ electrons, $G_{c c}^{\sigma}=\left\langle\left\langle c_{\mathbf{k} \sigma} ; c_{\mathbf{k} \sigma}^{\dagger}\right\rangle\right\rangle$ for itinerant $c$ electrons, and $G_{c \alpha}^{\sigma}=\left\langle\left\langle c_{\sigma} ; f_{\alpha \sigma}^{\dagger}\right\rangle\right\rangle$ and $G_{\alpha c}^{\sigma}=\left\langle\left\langle f_{\alpha \sigma} ; c_{\sigma}^{\dagger}\right\rangle\right\rangle$ for the "mixed" $c f$ states. As it is easier to solve the self-consistent system of equations in the momentum space, we evaluated all Green's functions in $\mathbf{k}$ representation. The equations of motion for these four Green's functions are given by

$$
\begin{gathered}
\left(\omega-E_{0 \sigma}\right) F_{\alpha \alpha}^{\sigma}(\mathbf{k})=1+\alpha_{\bar{\sigma}} G_{c \alpha}^{\sigma}(\mathbf{k}), \\
\left(\omega-E_{0 \sigma}\right) F_{\beta \alpha}^{\sigma}(\mathbf{k})=\alpha_{\bar{\sigma}} G_{c \alpha}^{\sigma}(\mathbf{k}), \\
\left(\omega-\varepsilon_{\mathbf{k} \sigma}\right) G_{c \alpha}^{\sigma}(\mathbf{k})=\alpha_{\bar{\sigma}}\left(F_{\alpha \alpha}^{\sigma}(\mathbf{k})+F_{\beta \alpha}^{\sigma}(\mathbf{k})\right), \\
\left(\omega-\varepsilon_{\mathbf{k} \sigma}\right) G_{c c}^{\sigma}(\mathbf{k})=1+\alpha_{\bar{\sigma}}\left(G_{\alpha c}^{\sigma}(\mathbf{k})+G_{\beta c}^{\sigma}(\mathbf{k})\right) .
\end{gathered}
$$

From equations of motion [Eqs. (9)], by straightforward but nevertheless tedious algebra (which we report in the Appendix) we obtain the following expressions for the Green's functions:

$$
\begin{aligned}
F_{\alpha \alpha}^{\sigma}(\mathbf{k})= & \frac{1}{2\left(\omega-E_{0 \sigma}\right)}-\frac{1}{2 W_{\sigma}(\mathbf{k})}\left[\frac{\varepsilon_{\mathbf{k} \sigma}-E_{+}^{\sigma}(\mathbf{k})}{\omega-E_{+}^{\sigma}(\mathbf{k})}\right. \\
& \left.-\frac{\varepsilon_{\mathbf{k} \sigma}-E_{-}^{\sigma}(\mathbf{k})}{\omega-E_{-}^{\sigma}(\mathbf{k})}\right], \\
F_{\beta \alpha}^{\sigma}(\mathbf{k})= & -\frac{1}{2\left(\omega-E_{0 \sigma}\right)}-\frac{1}{2 W_{\sigma}(\mathbf{k})}\left[\frac{\varepsilon_{\mathbf{k} \sigma}-E_{+}^{\sigma}(\mathbf{k})}{\omega-E_{+}^{\sigma}(\mathbf{k})}\right. \\
& \left.-\frac{\varepsilon_{\mathbf{k} \sigma}-E_{-}^{\sigma}(\mathbf{k})}{\omega-E_{-}^{\sigma}(\mathbf{k})}\right], \\
G_{c \alpha}^{\sigma}(\mathbf{k})= & \frac{\alpha_{\bar{\sigma}}}{W_{\sigma}(\mathbf{k})}\left[\frac{1}{\omega-E_{+}^{\sigma}(\mathbf{k})}-\frac{1}{\omega-E_{-}^{\sigma}(\mathbf{k})}\right], \\
G_{c c}^{\sigma}(\mathbf{k})=- & \frac{1}{W_{\sigma}(\mathbf{k})}\left[\frac{E_{0 \sigma}-E_{+}^{\sigma}(\mathbf{k})}{\omega-E_{+}^{\sigma}(\mathbf{k})}-\frac{E_{0 \sigma}-E_{-}^{\sigma}(\mathbf{k})}{\omega-E_{-}^{\sigma}(\mathbf{k})}\right],
\end{aligned}
$$

where $E_{ \pm}^{\sigma}(\mathbf{k})$ are given by Eq. (8) and 


$$
W_{\sigma}(\mathbf{k}) \equiv E_{+}^{\sigma}(\mathbf{k})-E_{-}^{\sigma}(\mathbf{k})=\sqrt{\left(E_{0 \sigma}-\varepsilon_{\mathbf{k} \sigma}\right)^{2}+8 \alpha_{\bar{\sigma}}^{2}} .
$$

The Green's functions given by Eqs. (10) will be evaluated self-consistently in the next subsection.

\section{Self-consistent equations}

We next construct a close self-consistent scheme to evaluate the bosonic fields $\lambda_{\uparrow}, \lambda_{\downarrow}, M$, and $m$ together with the chemical potential $\mu$ and the Lagrange multiplier $E_{0}$. This can be done by imposing (i) constraints on the total number of $f$ electrons, $n_{f}=2$, and $c$ electrons, $n_{c}$, correspondingly, (ii) the relation between the Fermi surface volume and the number of particles, (iii) the relation between the total magnetization and the number of electrons, and (iv) self-consistent equations for $\lambda_{\uparrow}$ and $\lambda_{\downarrow}$.

The numbers $n_{f}$ and $n_{c}$ are expressed via the integrals of the imaginary parts of the corresponding Green's functions as follows:

$$
\begin{aligned}
& n_{f}^{\sigma}=\int_{E_{\min }}^{\mu} d \omega \sum_{\mathbf{k} \alpha}\left[-\frac{1}{\pi} \operatorname{Im} F_{\alpha \alpha}^{\sigma}(\mathbf{k})\right], \\
& n_{c}^{\sigma}=\int_{E_{\min }}^{\mu} d \omega \sum_{\mathbf{k}}\left[-\frac{1}{\pi} \operatorname{Im} G_{c c}^{\sigma}(\mathbf{k})\right],
\end{aligned}
$$

where the summation is over all $k$ points of the Brillouin zone and the orbital indices $\alpha=1,2$. As the $k$ dependence in Eqs. (8) and (12) comes only through the bare conductionband energies $\varepsilon_{\mathbf{k} \sigma}$, we can substitute the summation in the $k$ space by the integration over $\varepsilon_{\sigma}$. The integration is over the interval $\left[-D+\Delta_{\sigma}, D+\Delta_{\sigma}\right]$ where the density of states $\rho_{0}$ $=\frac{1}{2 D}$ is a constant.

We first consider the case $T=0$ when the calculations can be done analytically. Let us first evaluate the number of $f$ electrons. For each polarization of spin, $f$ electrons can occupy one localized level $E_{0 \sigma}$ and a fraction of the two hybridized bands $E_{ \pm}^{\sigma}(\mathbf{k})$. The expression for spin-up $n_{f}^{\uparrow}$ can be then written as

$$
\begin{aligned}
n_{f}^{\uparrow}= & 1+\frac{1}{2 D} \int_{-D+\Delta_{\uparrow}}^{D+\Delta_{\uparrow}} \frac{\varepsilon_{\uparrow}-E_{-}^{\uparrow}\left(\varepsilon_{\uparrow}\right)}{W_{\uparrow}\left(\varepsilon_{\uparrow}\right)} d \varepsilon_{\uparrow} \\
& -\frac{1}{2 D} \int_{-D+\Delta_{\uparrow}}^{\mu_{\uparrow}+\Delta_{\uparrow}} \frac{\varepsilon_{\uparrow}-E_{+}^{\uparrow}\left(\varepsilon_{\uparrow}\right)}{W_{\uparrow}\left(\varepsilon_{\uparrow}\right)} d \varepsilon_{\uparrow}=\frac{7}{4}+\frac{\mu_{\uparrow}}{4 D} \\
& +\frac{1}{4 D} \int_{\mu_{\uparrow}+\Delta_{\uparrow}}^{D+\Delta_{\uparrow}} \frac{\varepsilon_{\uparrow}-E_{0 \uparrow}}{W_{\uparrow}\left(\varepsilon_{\uparrow}\right)} d \varepsilon_{\uparrow} .
\end{aligned}
$$

All parameters in Eq. (13) were defined in Eqs. (7) and (11). We have also used the following relations:

$$
\begin{aligned}
& \varepsilon_{\uparrow}-E_{-}^{\uparrow}\left(\varepsilon_{\uparrow}\right) \equiv \frac{1}{2}\left(\varepsilon_{\uparrow}-E_{0 \uparrow}+W_{\uparrow}\left(\varepsilon_{\uparrow}\right)\right), \\
& \varepsilon_{\uparrow}-E_{+}^{\uparrow}\left(\varepsilon_{\uparrow}\right) \equiv \frac{1}{2}\left(\varepsilon_{\uparrow}-E_{0 \uparrow}-W_{\uparrow}\left(\varepsilon_{\uparrow}\right)\right) .
\end{aligned}
$$

Taking into account that $\int_{a}^{b} \frac{x d x}{\sqrt{x^{2}+c}}=\left.\sqrt{x^{2}+c}\right|_{a} ^{b}$, we obtain

$$
\begin{aligned}
n_{f}^{\uparrow}= & \frac{7}{4}+\frac{\mu_{\uparrow}}{4 D}+\frac{1}{4 D}\left[\sqrt{\left(D+\Delta_{\uparrow}-E_{0 \uparrow}\right)^{2}+8 \alpha_{\downarrow}^{2}}\right. \\
& \left.-\sqrt{\left(\mu_{\uparrow}+\Delta_{\uparrow}-E_{0 \uparrow}\right)^{2}+8 \alpha_{\downarrow}^{2}}\right] .
\end{aligned}
$$

The quantity $\mu_{\uparrow}$, present in Eqs. (13)-(15) as the limit of integration over $\epsilon_{\uparrow}$, is related to $\mu$ through the relation $E_{+}^{\uparrow}\left(\mu_{\uparrow}\right)=\mu$.

By the same procedure we obtain the expressions for $n_{f}^{\downarrow}$, $n_{c}^{\uparrow}$, and $n_{c}^{\downarrow}$ as follows:

$$
\begin{aligned}
n_{f}^{\downarrow}= & \frac{1}{4}+\frac{\mu_{\downarrow}}{4 D}-\frac{1}{4 D}\left[\sqrt{\left(-D+\Delta_{\downarrow}-E_{0 \downarrow}\right)^{2}+8 \alpha_{\uparrow}^{2}}\right. \\
& \left.-\sqrt{\left(\mu_{\downarrow}+\Delta_{\downarrow}-E_{0 \downarrow}\right)^{2}+8 \alpha_{\uparrow}^{2}}\right], \\
n_{c}^{\uparrow}= & \frac{3}{4}+\frac{\mu_{\uparrow}}{4 D}-\frac{1}{4 D}\left[\sqrt{\left(D+\Delta_{\uparrow}-E_{0 \uparrow}\right)^{2}+8 \alpha_{\downarrow}^{2}}\right. \\
& \left.-\sqrt{\left(\mu_{\uparrow}+\Delta_{\uparrow}-E_{0 \uparrow}\right)^{2}+8 \alpha_{\downarrow}^{2}}\right], \\
n_{c}^{\downarrow}= & \frac{1}{4}+\frac{\mu_{\downarrow}}{4 D}-\frac{1}{4 D}\left[\sqrt{\left(\mu_{\downarrow}+\Delta_{\downarrow}-E_{0 \downarrow}\right)^{2}+8 \alpha_{\uparrow}^{2}}\right. \\
& \left.-\sqrt{\left(-D+\Delta_{\downarrow}-E_{0 \downarrow}\right)^{2}+8 \alpha_{\uparrow}^{2}}\right],
\end{aligned}
$$

where $\mu_{\downarrow}$ is obtained from $E_{-}^{\downarrow}\left(\mu_{\downarrow}\right)=\mu$.

Now we can construct the system of self-consistent equations. The first one is the constraint on the total number of $f$ electrons,

$$
n_{f}=n_{f}^{\uparrow}+n_{f}^{\downarrow}=2 .
$$

As we already discussed above, we replaced the local constraint $n_{f i}=2$ at each site $i$ by a softer one, for the average $n_{f}$.

The second self-consistent equation is obtained by setting the average number of conduction electrons to be equal to the filling value $n_{c}$ as follows:

$$
n_{c}=n_{c}^{\uparrow}+n_{c}^{\downarrow} .
$$

The third and fourth self-consistent equations are obtained from the Luttinger theorem, and from the condition that the total magnetization $M_{\text {tot }}$ is the sum of average magnetization of $f$ and $c$ electrons. This gives

$$
n_{f}+n_{c}=3+\frac{\mu_{\uparrow}+\mu_{\downarrow}}{2 D}
$$

and

$$
M+m=1+\frac{\mu_{\uparrow}-\mu_{\downarrow}}{4 D} .
$$

We used here $M=\frac{1}{2}\left(n_{f}^{\uparrow}-n_{f}^{\downarrow}\right)$ and $m=\frac{1}{2}\left(n_{c}^{\uparrow}-n_{c}^{\downarrow}\right)$, respectively. We remark that the Fermi surface encloses both conduction electrons and partially localized $f$ levels.

The last two equations are self-consistent relations for the bosonic fields $\lambda_{\uparrow}, \lambda_{\downarrow}$. These two fields are related to the imaginary part of the mixed $c f$ Green's function as follows:

$$
\lambda_{\sigma}=\int_{E_{\min }}^{\mu} d \omega \sum_{\mathbf{k}}\left[-\frac{1}{\pi}\left(\operatorname{Im} G_{c 1}^{\sigma}(\mathbf{k})+\operatorname{Im} G_{c 2}^{\sigma}(\mathbf{k})\right)\right] .
$$

Using Eq. (10) we then obtain the following expressions for $\lambda_{\sigma}$ : 


$$
\begin{gathered}
\lambda_{\uparrow}=-2 \frac{\alpha_{\downarrow}}{2 D}\left[\int_{-D+\Delta_{\uparrow}}^{D+\Delta_{\uparrow}} \frac{d \varepsilon_{\uparrow}}{W_{\uparrow}\left(\varepsilon_{\uparrow}\right)}-\int_{-D+\Delta_{\uparrow}}^{\mu_{\uparrow}+\Delta_{\uparrow}} \frac{d \varepsilon_{\uparrow}}{W_{\uparrow}\left(\varepsilon_{\uparrow}\right)}\right]= \\
-\frac{\alpha_{\downarrow}}{D} \int_{\mu_{\uparrow}+\Delta_{\uparrow}}^{D+\Delta_{\uparrow}} \frac{d \varepsilon_{\uparrow}}{W_{\uparrow}\left(\varepsilon_{\uparrow}\right)}, \\
\lambda_{\downarrow}=-\frac{\alpha_{\downarrow}}{D} \int_{-D+\Delta_{\downarrow}}^{\mu_{\downarrow}+\Delta_{\downarrow}} \frac{d \varepsilon_{\downarrow}}{W_{\downarrow}\left(\varepsilon_{\downarrow}\right)} .
\end{gathered}
$$

Using $\int \frac{d x}{\sqrt{x^{2}+c}}=\ln \left(x+\sqrt{x^{2}+c}\right)$ we find

$$
\begin{gathered}
\lambda_{\uparrow}=-\frac{\alpha_{\downarrow}}{D} \ln \frac{D+\Delta_{\uparrow}-E_{0 \uparrow}+W_{\uparrow}\left(D+\Delta_{\uparrow}\right)}{\mu_{\uparrow}+\Delta_{\uparrow}-E_{0 \uparrow}+W_{\uparrow}\left(\mu_{\uparrow}+\Delta_{\uparrow}\right)}, \\
\lambda_{\downarrow}=-\frac{\alpha_{\uparrow}}{D} \ln \frac{\mu_{\downarrow}+\Delta_{\downarrow}-E_{0 \downarrow}+W_{\downarrow}\left(\mu_{\downarrow}+\Delta_{\downarrow}\right)}{-D+\Delta_{\downarrow}-E_{0 \downarrow}+W_{\downarrow}\left(-D+\Delta_{\downarrow}\right)} .
\end{gathered}
$$

Equations (17)-(20) and (23) constitute the full set of self-consistent equations for six variables: $\lambda_{\uparrow}, \lambda_{\downarrow}, M, m, \mu$, and $E_{0}$. We solved this equations numerically by iteration and we explicitly verified that the solution minimizes the total internal energy $E_{\text {tot }}$

$$
\begin{aligned}
E_{\mathrm{tot}}= & \frac{7}{4} E_{0 \uparrow}+\frac{1}{4 D}\left[\mu_{\uparrow} E_{0 \uparrow}+\left(\mu_{\downarrow}+D\right) E_{0 \downarrow}-D^{2}+2 D\left(\Delta_{\uparrow}-\Delta_{\downarrow}\right)\right. \\
& \left.+\frac{\mu_{\uparrow}^{2}+\mu_{\downarrow}^{2}}{2}+\Delta_{\uparrow} \mu_{\uparrow}+\Delta_{\downarrow} \mu_{\downarrow}\right]-\frac{1}{8 D}\left[\left(D+\Delta_{\uparrow}-E_{0 \uparrow}\right) W_{\uparrow}(D\right. \\
& \left.+\Delta_{\uparrow}\right)-\left(\mu_{\uparrow}+\Delta_{\uparrow}-E_{0 \uparrow}\right) W_{\uparrow}\left(\mu_{\uparrow}+\Delta_{\uparrow}\right)+\left(D+\Delta_{\uparrow}\right. \\
& \left.\left.+E_{0 \downarrow}\right) W_{\downarrow}\left(-D+\Delta_{\downarrow}\right)+\left(\mu_{\downarrow}+\Delta_{\downarrow}-E_{0 \downarrow}\right) W_{\downarrow}\left(\mu_{\downarrow}+\Delta_{\downarrow}\right)\right] \\
& +J_{K} \lambda_{\downarrow} \lambda_{\uparrow}-J_{K} m M-\frac{1}{2} J_{H} z M^{2}-E_{F} n_{c}-2 E_{0} .
\end{aligned}
$$

At finite temperature, $T$, the set of self-consistent equations remains the same; however, we are no longer able to obtain an analytical expression for the occupation numbers and bosonic fields. The relations between $n_{f}^{\sigma}, n_{c}^{\sigma}, \lambda_{\sigma}$, and the Green's functions are now

$$
\begin{gathered}
n_{f}^{\sigma}=\int_{-\infty}^{+\infty} d \omega n_{F}(\omega) \sum_{\mathbf{k}}\left[-\frac{1}{\pi} \operatorname{Im}\left(F_{11}^{\sigma}(\mathbf{k})+F_{22}^{\sigma}(\mathbf{k})\right)\right], \\
n_{c}^{\sigma}=\int_{-\infty}^{+\infty} d \omega n_{F}(\omega) \sum_{\mathbf{k}}\left[-\frac{1}{\pi} \operatorname{Im} G_{c c}^{\sigma}(\mathbf{k})\right], \\
\lambda_{\sigma}=\int_{-\infty}^{+\infty} d \omega n_{F}(\omega) \sum_{\mathbf{k}}\left[-\frac{1}{\pi}\left(\operatorname{Im} G_{c 1}^{\sigma}(\mathbf{k})+\operatorname{Im} G_{c 2}^{\sigma}(\mathbf{k})\right)\right],
\end{gathered}
$$

where $n_{F}(\omega)=\frac{1}{e^{\omega-\mu / T}+1}$ is the Fermi distribution function. Straightforward calculations lead to

$$
\begin{aligned}
n_{f}^{\sigma}= & \frac{1}{2 D} \int_{-D+\Delta_{\sigma}}^{D+\Delta_{\sigma}} d \varepsilon_{\sigma}\left[n_{F}\left(E_{0 \sigma}\right)-n_{F}\left(E_{+}^{\sigma}\left(\varepsilon_{\sigma}\right)\right) \frac{\varepsilon_{\sigma}-E_{+}^{\sigma}\left(\varepsilon_{\sigma}\right)}{W_{\sigma}\left(\varepsilon_{\sigma}\right)}\right. \\
& \left.+n_{F}\left(E_{-}^{\sigma}\left(\varepsilon_{\sigma}\right)\right) \frac{\varepsilon_{\sigma}-E_{-}^{\sigma}\left(\varepsilon_{\sigma}\right)}{W_{\sigma}\left(\varepsilon_{\sigma}\right)}\right] \\
n_{c}^{\sigma}= & \frac{1}{2 D} \int_{-D+\Delta_{\sigma}}^{D+\Delta_{\sigma}} d \varepsilon_{\sigma}\left[-n_{F}\left(E_{+}^{\sigma}\left(\varepsilon_{\sigma}\right)\right) \frac{E_{0 \sigma}-E_{+}^{\sigma}\left(\varepsilon_{\sigma}\right)}{W_{\sigma}\left(\varepsilon_{\sigma}\right)}\right. \\
& \left.+n_{F}\left(E_{-}^{\sigma}\left(\varepsilon_{\sigma}\right)\right) \frac{E_{0 \sigma}-E_{-}^{\sigma}\left(\varepsilon_{\sigma}\right)}{W_{\sigma}\left(\varepsilon_{\sigma}\right)}\right], \\
\lambda_{\sigma}= & \frac{1}{D} \int_{-D+\Delta_{\sigma}}^{D+\Delta_{\sigma}} d \varepsilon_{\sigma}\left[n_{F}\left(E_{+}^{\sigma}\left(\varepsilon_{\sigma}\right)\right)-n_{F}\left(E_{-}^{\sigma}\left(\varepsilon_{\sigma}\right)\right)\right] \frac{\alpha_{\bar{\sigma}}}{W_{\sigma}\left(\varepsilon_{\sigma}\right)} .
\end{aligned}
$$

As for $T=0$, we now evaluate numerically the integrals in Eqs. (26) and verified that the numerical solution of selfconsistent equations minimizes the free energy of the system $F$. The latter can be calculated through the partition function $Z$ as follows:

$$
Z=\sum_{\mathbf{k} \sigma}\left[e^{-\beta\left(E_{-}^{\sigma}(\mathbf{k})-\mu\right)}+e^{-\beta\left(E_{+}^{\sigma}(\mathbf{k})-\mu\right)}\right]+\sum_{\sigma} e^{-\beta\left(E_{0 \sigma^{-}} \mu\right)}
$$

and

$$
F=-T \ln Z .
$$

In the following section, we present the detailed discussion of the results obtained with this self-consistent scheme for the UKL model.

\section{RESULTS}

\section{A. $T=0$}

We first discuss the properties of the model at $T=0$. In order to establish the region of coexistence of Kondo effect and ferromagnetic ordering (Kondo-ferromagnetism coexistence) in the phase space set by the parameters of the model, we study the behavior of $\lambda_{\sigma}$ and the magnetization $M$ and $m$. These order parameters are correlated and it is useful to discuss them together. In Fig. 2 we present the variation of the Kondo correlation $\lambda_{\uparrow}$ as a function of the Kondo coupling, $J_{K}$, and the band filling, $n_{c}$, for a fixed value of $J_{H}=-0.01$. In Fig. 3 we present the dependence of the total magnetization $(M+m)$ on the same parameters. In these and all other figures all energies and temperatures are measured in units of the half-bandwidth $D$. The values of $\lambda_{\downarrow}$ and $\lambda_{\uparrow}$ are close; therefore, we present just $\lambda_{\uparrow}$ in Fig. 2 in order to keep the figure transparent.

From the analysis of both figures one can verify that for values of $J_{K}$ smaller than 0.6 , the Kondo correlation $\lambda \uparrow$ remains equal to zero (Fig. 2) for all values of $n_{c}$ considered. The total magnetization $M+m$ is large and equal to its maximum value (Fig. 3). This phase is a pure magnetic one with no Kondo effect. When $J_{K}$ increases, for a fixed value of $n_{c}$, there exists a critical value of $J_{K}$ above which both $\lambda_{\uparrow}$ and the total magnetization $M_{\text {tot }}$ change abruptly and $\lambda_{\uparrow}$ become 


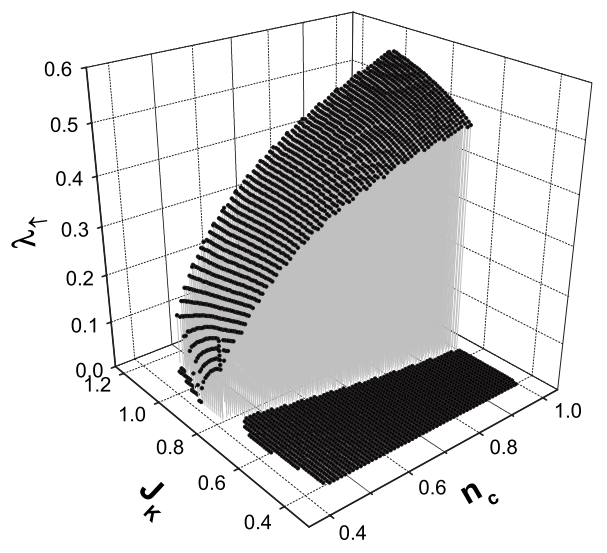

FIG. 2. Plot of the parameter $\lambda_{\uparrow}$ as a function of $J_{K}$ and $n_{c}$ for $J_{H}=-0.01$. There is a discontinuous transition as a function of $J_{K}$ and a behavior in $n_{c}^{1 / 2}$ as explained in the text.

different from zero. The region of the parameters $J_{K}$ and $n_{c}$ where $\lambda_{\uparrow}$ and the total magnetization $M_{\text {tot }}$ are finite is the region of coexistence between Kondo effect and ferromagnetic order. As the magnetization of localized and conduction electrons, $M$ and $m$, have opposite signs due to antiferromagnetic Kondo coupling between them, the maximum value of the total magnetization is always less than 1 . The critical value of $J_{K}$ decreases with increasing $n_{c}$, as it is expected from the "exhaustion principle." $3,35,37$

One can see in Fig. 2 that $\lambda_{\uparrow}$ decreases smoothly as a function of $n_{c}$, while it undergoes a sharp transition as a function of $J_{K}$. We calculated the variation of $\lambda$ with $n_{c}$ and obtained an approximate square root behavior $\lambda \sim\left(n_{c}\right.$ $\left.-n_{c, c r}\right)^{r}$, with $r \approx 1 / 2$, similar to the KL case with spin 1/2. ${ }^{3,35}$ Indeed, in Fig. 4 we present a plot of $\lambda_{\uparrow}$ as a function of $n_{c}$ for $J_{K}=0.9$ and $J_{H}=-0.01$. The solid line in this figure is the best fit to the numerical results and yields a power law behavior with an exponent very near $1 / 2$.

We next study the effect of the exchange interaction between localized spins, $J_{H}$, on the region of the Kondoferromagnetism coexistence. In Fig. 5 we present the phase diagram in the $\left\{J_{K}, J_{H}\right\}$ plane for a fixed value of $n_{c}$. The

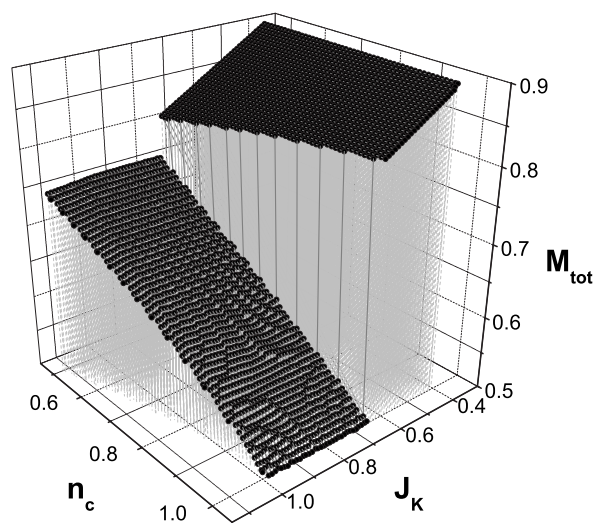

FIG. 3. Plot of the total magnetization $(M+m)$ as a function of $J_{K}$ and $n_{c}$ for $J_{H}=-0.01$. There is a discontinuous transition as a function of $J_{K}$ that corresponds to the transition to $\lambda_{\uparrow}=0$ in Fig. 2.

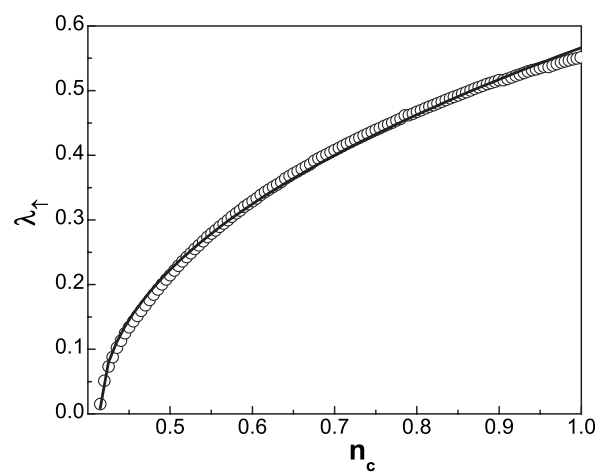

FIG. 4. Plot of the parameter $\lambda_{\uparrow}$ as a function of $n_{c}$, for $J_{K}$ $=0.9$ and $J_{H}=-0.01$. The circles correspond to the numerical calculation and the line to a fit with a square root law. $\lambda_{\uparrow}$ goes to zero for $n_{c} \simeq 0.42$.

region of coexistence extends up to $J_{K}=1.2$ and down to $J_{K}=0.5$ while $J_{H}$ can vary between -0.001 and -0.06 . The values of $J_{H}$ in Fig. 5 are rather small; however, we recall that the real strength of the "local field" applied on an individual spin by its neighbors is $z J_{H}$, where $z=6$ is the number of nearest neighbors in a simple cubic lattice.

Summarizing, at $T=0$ there exists a region of coexistence between the Kondo effect and the ferromagnetic order in a wide range of parameters $J_{K}, J_{H}$, and $n_{c}$, including the case of the half-filled conduction band, $n_{c}=1$. Also, a discontinuous transition to pure ferromagnetic state is found by decreasing $J_{K}$ while a continuous change is obtained varying the band filling, $n_{c}$.

\section{B. Finite temperatures}

We now present the results obtained at finite temperatures. First we show in Fig. 6 the temperature variation of the Kondo correlations $\lambda_{\uparrow}$ and $\lambda_{\downarrow}$, and also the $f$ and $c$ magnetization $M$ and $m$. The parameters used in the calculation of Fig. 6 are $J_{K}=0.8, J_{H}=-0.01$, and $n_{c}=0.8$. The two magne-

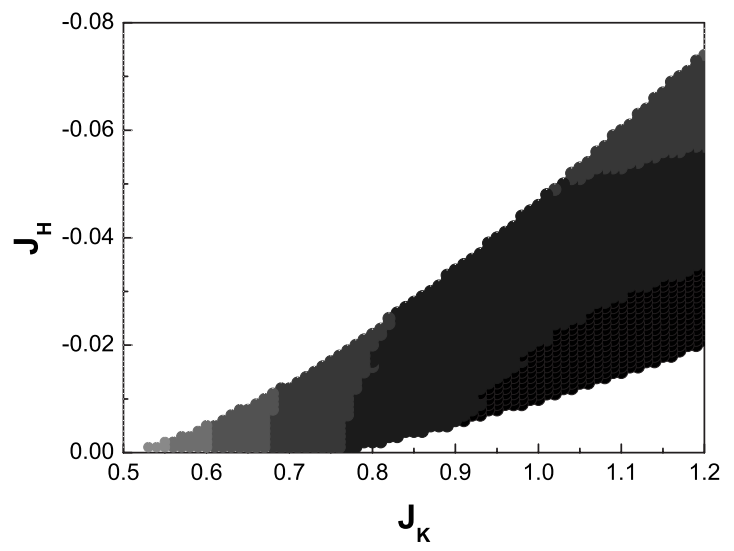

FIG. 5. The dark region indicates the values of the parameters $J_{K}$ and $J_{H}$ where there is coexistence between the magnetic ordered state and the strongly correlated (Kondo) state. The intensity of the gray tone is proportional to the value of $\lambda_{\uparrow}$. 


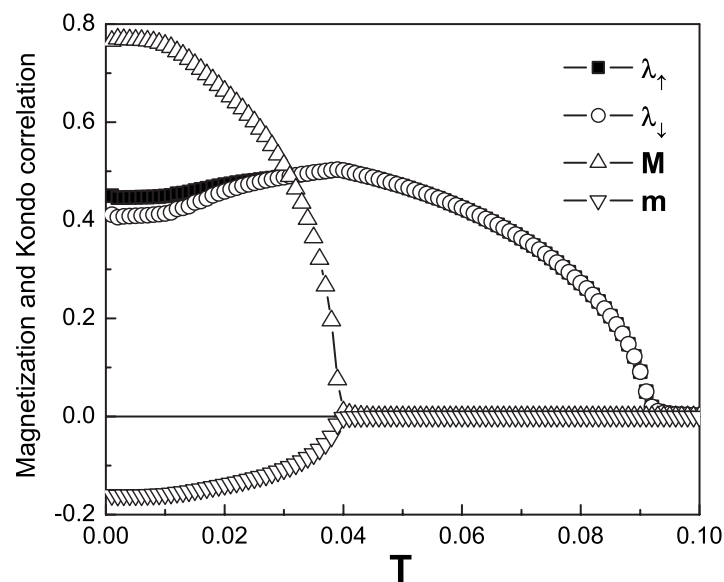

FIG. 6. Plot of $\lambda_{\uparrow}, \lambda_{\downarrow}, M$, and $m$ as a function of temperature for $J_{K}=0.8, J_{H}=-0.01$, and $n_{c}=0.8$. At zero and low temperatures we observe the coexistence of magnetic order and heavy fermion behavior; the Kondo effect is maximal when magnetization vanishes.

tization curves clearly show the existence of a continuous phase transition at the Curie, $T_{C}$, from a ferromagnetic to a paramagnetic state. At low temperatures we observe the coexistence of a magnetic order and Kondo behavior. The strength of the Kondo effect is the highest at $T_{C}$. As a matter of fact, when the magnetization decreases, $\lambda_{\sigma}$ grows and passes through a maximum at the $T_{C}$; its value at that temperature is about $20 \%$ bigger than at $T=0$. Due to the breakdown of the spin symmetry, $\lambda_{\downarrow}$ and $\lambda_{\uparrow}$ are slightly different in the magnetic region but they coincide at $T_{C}$, when the spin symmetry is restored. For $T>T_{C}$ the system exhibits only Kondo behavior $\left(\lambda_{\sigma} \neq 0, M=0\right.$, and $\left.m=0\right)$. Finally, we define the temperature at which $\lambda_{\sigma}$ vanishes and $f$ and $c$ electrons become decoupled, as the Kondo temperature, $T_{K}$. The fact that $\lambda$ vanishes at a particular temperature, instead of slowly decaying to zero, is a well-known artifact of the mean-field approximation. Actually, $T_{K}$ is a crossover temperature, associated with the onset of local Kondo screening.

\section{Density of states}

We also analyze the $f$ and $c$ electron densities of states (DOSs) at various temperatures. The $f$ and $c$ electron DOSs are calculated numerically from the imaginary part of the $f-f$ and $c-c$ Green's functions.

$$
\begin{aligned}
& \rho_{f}(\omega)=\sum_{k \alpha}\left(-\frac{1}{\pi} \operatorname{Im} F_{\alpha \alpha}^{\sigma}\right), \\
& \rho_{c}(\omega)=\sum_{k}\left(-\frac{1}{\pi} \operatorname{Im} G_{c c}^{\sigma}\right) .
\end{aligned}
$$

Figure 7 shows the $f$ and $c$ DOSs at $T=0$. The parameters are the same as in Fig. 6. For these parameters, both the magnetic order and the Kondo effect are present in the ground state. We see from the figure that the bands for the two spin polarizations are shifted, as expected for a magnetically ordered state. The hybridization gap due to Kondo ef-

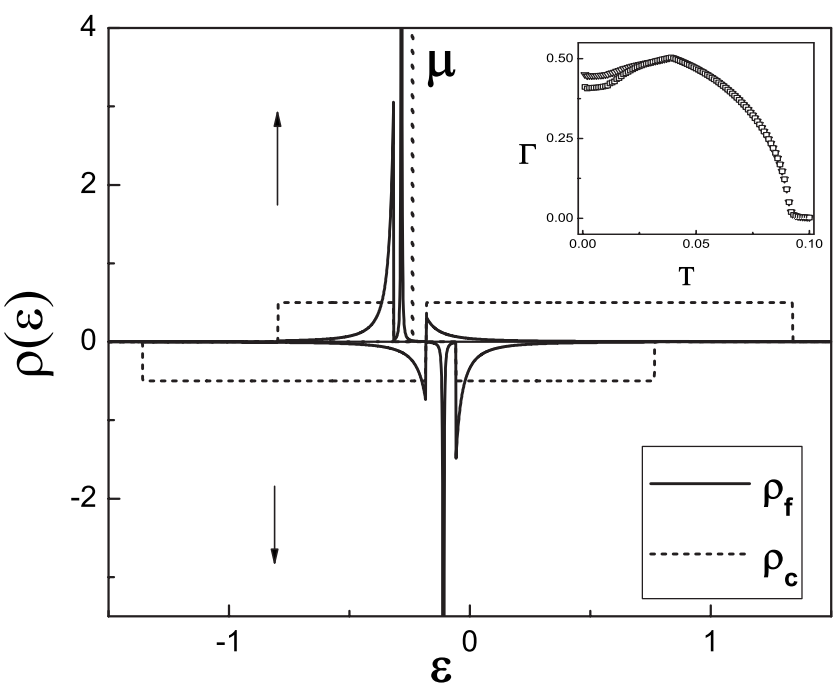

FIG. 7. Schematic plot of the density of states for the magnetic case at $T=0$. The parameters are $J_{K}=0.8, J_{H}=-0.01$, and $n_{c}=0.8$. The continuous line corresponds to the $f$ density of states, the dashed line to the conduction density of states, and the dotted vertical line indicates the position of the Fermi energy. The peak of the localized $f$ level lies inside the gap and here the up localized $f$ level is occupied and the down localized $f$ level is empty. The inset shows the variation of the hybridization gap for the two spin directions as a function of the temperature.

fect is present for both directions of the spin, but they do not coincide neither in the borders nor in the width. The nonhybridized $f$ level lies inside this gap. This localized $f$ level is occupied for spin-up states and it is empty for spin-down states. We caution that the finite width for the two localized $f$ levels in the figure is due to the fact that we added a very small but finite imaginary part to the energies. The inset of Fig. 7 shows the temperature variations of the hybridization gaps for two spin directions. The two gaps are nearly equal because they are proportional to $\lambda_{\sigma}$, and the difference between $\lambda_{\uparrow}$ and $\lambda_{\downarrow}$ is rather small.

In Fig. 8 we plot the density of states at four different temperatures: (a) $T=0$ and (b) a low-temperature phase ( $T$ $<T_{C}$ ) both exhibiting coexistence of magnetic order and Kondo behavior, (c) pure Kondo phase at $T_{C}<T<T_{K}$, and (d) uncorrelated high-temperature phase at $T>T_{K}$. In the coexistence region at $T<T_{C}$, the Fermi level remains inside the hybridization gap for the spin-up band, and inside the conduction band, $E_{-}^{\downarrow}(k)$, for the spin-down band. That implies a semimetallic behavior. When $T>T_{C}$, the up- and down-spin bands coincide, and the Fermi level lies inside the gap and coincides with the energy of the $f$ level, $E_{0 \sigma}$. That implies an insulating behavior. Finally, when $T>T_{K}, \lambda_{\sigma}$ vanishes and there is no more coupling between $f$ and $c$ electrons: the hybridization gap closes and the system becomes metallic.

\section{Effective mass}

From the quasiparticle spectrum (8) one can estimate the mass enhancement ${ }^{38}$ 


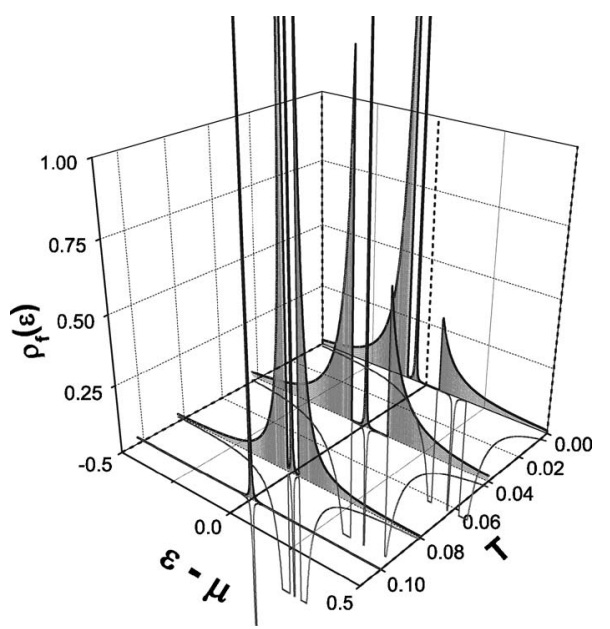

FIG. 8. Plot of the up and down $f$ densities of states for $T=0$ and three finite temperatures.

$$
\frac{m_{\sigma}^{*}}{m}=1+\frac{2 \alpha_{\bar{\sigma}}^{2}}{\left(E_{0 \sigma}-\mu-\Delta_{\sigma}\right)^{2}} .
$$

We plot the temperature variation of the mass enhancement in Fig. 9. Two peaks are clearly seen: one corresponds to the Curie temperature, and the second (at a higher temperature) to the onset of Kondo effect. In the region of coexistence, the effective mass increases as a function of temperature tracking the position of the Fermi level inside the $E_{-}^{\bar{\sigma}}(k)$ band. In the pure Kondo phase at $T_{C}<T<T_{K}$, the mass enhancement becomes quite large. Such a large enhancement is the consequence of the fact that the denominator in Eq. (31) goes to zero in the pure Kondo regime, and the effective mass formally diverges. A finite width of the $f$ level eliminates the divergence, but still yields a very large mass. Although this estimate of the mass probably overestimates the strength of the enhancement, it still provides a qualitatively good explanation of the heavy fermion behavior.

\section{E. Specific heat}

The thermodynamic properties of the UKL model can be easily calculated from the expression for the free energy [Eq.

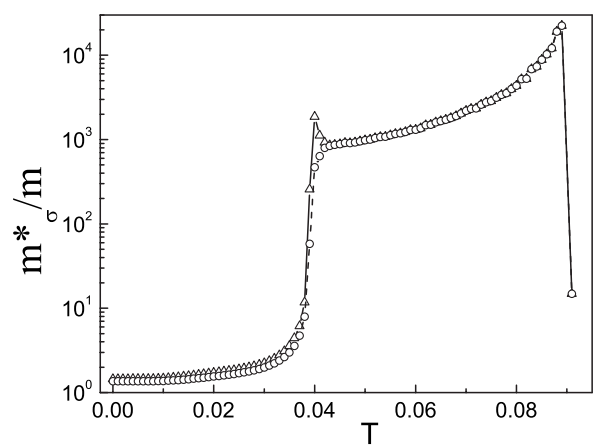

FIG. 9. Plot of the effective mass versus temperature with the same parameters as in Fig. 6. The triangles correspond to up spin and the circles to down spin.

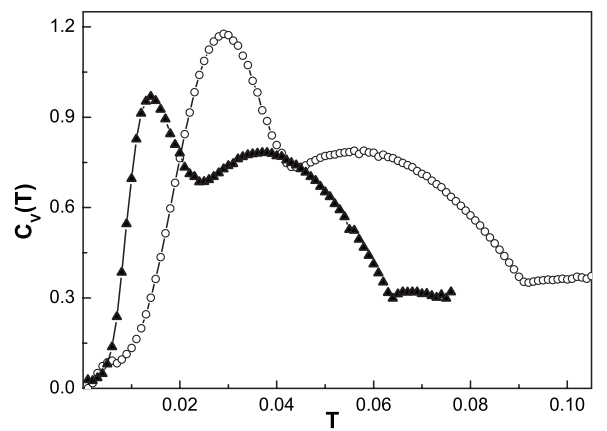

FIG. 10. Plot of the electronic specific heat for the parameters (a) $J_{K}=0.8, J_{H}=-0.01$, and $n_{c}=0.8$ (circles) and (b) $J_{K}=0.7, J_{H}=$ -0.001 , and $n_{c}=0.8$ (triangles).

(28)]. Here, we present only results for the specific heat which could be compared with available experimental data. We use the usual definition of the entropy of the system

$$
S(T)=-\frac{\partial F}{\partial T}=-\frac{\partial}{\partial T}(-T \ln Z)
$$

and the specific heat can be calculated as

$$
\begin{aligned}
C_{V}(T)= & T \frac{\partial S}{\partial T}=\frac{1}{T^{2}} \sum_{k, \sigma}\left[\frac{\left(E_{-}^{\sigma}(\mathbf{k})-\mu\right)^{2} e^{\beta\left(E_{-}^{\sigma}(\mathbf{k})-\mu\right)}}{\left(1+e^{\beta\left(E_{-}^{\sigma}(\mathbf{k})-\mu\right)}\right)^{2}}\right. \\
& \left.+\frac{\left(E_{+}^{\sigma}(\mathbf{k})-\mu\right)^{2} e^{\beta\left(E_{+}^{\sigma}(\mathbf{k})-\mu\right)}}{\left(1+e^{\beta\left(E_{+}^{\sigma}(\mathbf{k})-\mu\right)}\right)^{2}}\right] \\
& +\frac{1}{T^{2}} \sum_{\sigma} \frac{\left(E_{0 \sigma}-\mu\right)^{2} e^{\beta\left(E_{0 \sigma^{-}} \mu\right)}}{\left[1+e^{\beta\left(E_{0 \sigma^{-}}\right)}\right]^{2}} .
\end{aligned}
$$

In Fig. 10 we present numerical results for the specific heat as a function of temperature. As the specific heat is very sensitive to the choice of the parameters, we plot it for two different sets: (a) $J_{K}=0.8, J_{H}=-0.01, n_{c}=0.8$ (circles), and (b) for $J_{K}=0.7, J_{H}=-0.001, n_{c}=0.8$ (triangles). For the latter choice of parameters, the specific heat temperature dependence is more pronounced. First, at low temperatures, the specific heat increases with increasing $T$, following a power law with an exponent close to 2 . Second, it goes through a peak, which can be associated with the temperature when both $\lambda_{\uparrow}$ and $\lambda_{\downarrow}$ become equal and to the Curie temperature. At higher temperature, in the nonmagnetic region, the specific heat is very high because the Fermi level coincides with the localized $f$ level, as it was discussed for the effective mass. Those values are so high that they mask a second peak, expected for the Kondo temperature. Therefore, when the gap in the density of states closes at the Kondo temperature, only a change in the slope is observed. In case (a) both peaks are "rounded up" because of the higher values of the order parameters compared to case (b).

\section{F. Ferromagnetic Doniach diagram}

We present here the ferromagnetic Doniach diagram for the UKL model. In Fig. 11, we plot the Curie temperature, $T_{C}$, and the Kondo temperature, $T_{K}$, versus $J_{K}$ for fixed val- 


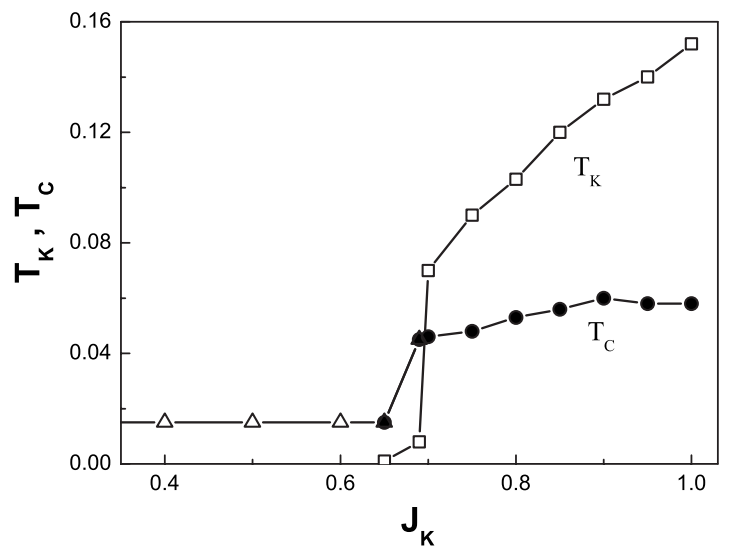

FIG. 11. The ferromagnetic Doniach diagram: Plot of the Curie temperature $T_{C}$ (the triangles are analytical values, and the circles numerical ones) and the Kondo temperature $T_{K}$ (squares) versus $J_{K}$ with $J_{H}=-0.01$ and $n_{c}=0.8$.

ues of $J_{H}$ and $n_{c}$. It is possible to see that the Kondo temperature $T_{K}$ becomes finite only at the critical value $J_{K}^{c}$ $\sim 0.65$ for $J_{H}=-0.01$ and $n_{c}=0.8$, then rapidly increases for larger values of $J_{K}$. On the other hand, the Curie temperature, $T_{C}$, is finite for all studied values of $J_{K}$. The two curves $T_{K}\left(J_{K}\right)$ and $T_{C}\left(J_{K}\right)$ cross slightly above $J_{K}^{c}$ and for larger values of $J_{K}$ the Kondo temperature, $T_{K}$, is always larger than $T_{C}$. Indeed, the ferromagnetic order persists for all values of the ratio $J_{K} / J_{H}$, while the Kondo-ferromagnetism coexistence exists only for sufficiently large values of this ratio.

In the purely magnetic region the Curie temperature can be easily evaluated, and it is equal to $T_{C}=\frac{z\left|J_{H}\right|}{4}$. Also, the Kondo temperature exhibits an almost linear behavior as a function of $J_{K}$. This is in contrast with the Kondo impurity case, where an exponential dependence on $J_{K}$ is observed, ${ }^{39}$ and also with the $S=1 / 2 \mathrm{KL}$ model where both exponential and nonexponential behaviors can be obtained. ${ }^{3}$

It is worth to note that the present results indicate that the Curie temperature increases as a function of $J_{K}$, and this is also in the opposite direction of the standard Doniach's diagram. This can be understood because in the Doniach's diagram (localized spins $S=1 / 2$ ), the reduction of the Néel temperature at large $J_{K}$ is the consequence of the competition between magnetic order and Kondo effect. The ordering temperature goes to zero at high enough values of $J_{K}$. Here, there is no such competition, and the Kondo interaction actually favors a ferromagnetic ordering through the RKKY interaction (even if the localized magnetic moments are partially screened by the conduction electrons). That explains the augmentation of the Curie temperature. Then, if in the standard KL model the short-range antiferromagnetic correlations between neighboring $f$ moments reinforce the Kondo effect, ${ }^{5,6}$ in the UKL model it is the Kondo effect that reinforces the ferromagnetic interaction.

The diagram presented in Fig. 11 can be called the "ferromagnetic Doniach diagram" for the UKL model and it is qualitatively very different from the well-known Doniach diagram derived for the Kondo lattice with $S=1 / 2$, where the ordering temperature tends to zero at a finite $J_{K}$ and the magnetic order and the Kondo effect compete rather than coexist.

\section{CONCLUSIONS}

In conclusion, in this work we introduced and studied the underscreened Kondo lattice model with localized spins $S$ $=1$. In the framework of this model, we analyzed the coexistence of ferromagnetism and Kondo behavior. To this end, we derived and solved a set of self-consistent equations for the relevant bosonic fields. We obtained a region in the space of parameters, where the order parameters, $\lambda_{\sigma}, M$, and $m$, are simultaneously different from zero. This coexistence persists up to finite temperatures, then the magnetic order disappears first, at the temperature $T_{C}$. At this temperature $\lambda_{\sigma}$ is still different from zero. In fact, it is at its maximum value, indicating that the Kondo effect is the strongest. This is, in fact, in agreement with experiments for uranium compounds in which the Kondo behavior is observed above the Curie temperature. The phase transition at $T_{C}$ is, therefore, a transition from the ferromagnetic Kondo phase at low $T$ to the nonmagnetic Kondo phase at higher $T$. Another transition can be obtained at $T=0$ by varying the Kondo coupling parameter $J_{K}$. This transition is discontinuous and leads to a non-Kondo magnetically ordered state (see Fig. 2).

We emphasize that the coexistence of ferromagnetism and Kondo effect is due to the presence of two $f$ levels: one nonhybridized and one hybridized levels (see Fig. 7). The presence of the nonhybridized $f$ level is at the origin of the partial screening of the localized spins. Indeed, this is the key difference between our UKL model and $S=1 / 2$ Kondo lattice model. In the latter, there is only one $f$ level and it is always hybridized with the conduction band in the Kondo regime. The result is a competition between magnetism and Kondo effect which is reflected in Doniach diagram.

Our UKL model can explain the behavior of some uranium compounds such as the previously described UTe, $\mathrm{UCu}_{0.9} \mathrm{Sb}_{2}$, and $\mathrm{UCo}_{0.5} \mathrm{Sb}_{2}$ compounds, which order ferromagnetically at a large Curie temperature, $T_{C}$, and present a Kondo behavior. Such a Kondo-ferromagnetism coexistence has been recently observed in other uranium compounds. We would like to mention $\mathrm{UNiSi}_{2}$ with a Curie temperature $T_{C}$ $=95 \mathrm{~K},{ }^{40-42} \quad \mathrm{UCo}_{0.6} \mathrm{Ni}_{0.4} \mathrm{Si}_{2}$ with $T_{C}=62 \mathrm{~K},{ }^{42,43}$ and $\mathrm{URu}_{2-x} \mathrm{Re}_{x} \mathrm{Si}_{2}$ compounds where $T_{C}$ increases rapidly with concentration $x .^{44-46}$ In these compounds $T_{C}$ increases rapidly with $x$ and there are clear evidences of the coexistence between the ferromagnetic order and a non-Fermi-liquid behavior. ${ }^{45}$ A more extended analysis of the UKL model can, possibly, describe also this phenomenum. Our UKL model can finally be applied to the case of the recently observed neptunium compound $\mathrm{NpNiSi}_{2}$, which becomes ferromagnetic at $T_{C}=51.5 \mathrm{~K}$ and presents Kondo behavior. ${ }^{47} \mathrm{In}$ the neptunium based compounds, $5 f$ electrons are relatively well localized and the magnetic moment of $\mathrm{Np}$ can be described by a localized spin larger than $S=1 / 2$, again corresponding to underscreened case.

In conclusion, the UKL is a very good tool to describe some ferromagnetic-Kondo compounds and can be considered as an improvement with respect to the regular Kondo lattice model to account for some actinide $5 f$ compounds. 


\section{ACKNOWLEDGMENTS}

N.B.P. thanks MPIPKS, Dresden, Germany, where this work has been partially performed and the hospitality and support of IF-UFRGS and FAPERGS, Porto Alegre, Brazil. B.C. thanks the European COST P16 for financial support. J.R.I. acknowledges the hospitality of MPIPKS, Dresden, Germany and LPS, UPS, Orsay, France and financial support from Brazilian agencies CNPq, CAPES, and FAPERGS.

\section{APPENDIX}

In this appendix we present the derivation of the Green's functions [Eqs. (10)] using the equations of motion [Eqs. (9)].

We first derive the Green's functions for the $f$ electrons as follows:

$$
\begin{gathered}
\left(\omega-E_{0 \sigma}\right) F_{\alpha \alpha}^{\sigma}(\mathbf{k})=1+\alpha_{\bar{\sigma}} \frac{\alpha_{\bar{\sigma}}\left(F_{\alpha \alpha}^{\sigma}(\mathbf{k})+F_{\beta \alpha}^{\sigma}(\mathbf{k})\right)}{\omega-\varepsilon_{\mathbf{k} \sigma}}, \\
\left(\omega-E_{0 \sigma}\right) F_{\beta \alpha}^{\sigma}(\mathbf{k})=\alpha_{\bar{\sigma}} \frac{\alpha_{\bar{\sigma}}\left(F_{\alpha \alpha}^{\sigma}(\mathbf{k})+F_{\alpha \alpha}^{\sigma}(\mathbf{k})\right)}{\omega-\varepsilon_{\mathbf{k} \sigma}},
\end{gathered}
$$

where we call $A_{\bar{\sigma}}=\frac{\alpha_{\bar{\sigma}}^{2}}{\omega-\varepsilon_{\mathbf{k} \sigma}}$. Then, Eq. (A1) becomes

$$
\begin{gathered}
\left(\omega-E_{0 \sigma}-A_{\bar{\sigma}}\right) F_{\alpha \alpha}^{\sigma}(\mathbf{k})=1+A_{\bar{\sigma}} F_{\beta \alpha}^{\sigma}(\mathbf{k}), \\
\left(\omega-E_{0 \sigma}\right) F_{\beta \alpha}^{\sigma}(\mathbf{k})=A_{\bar{\sigma}}\left(F_{\alpha \alpha}^{\sigma}(\mathbf{k})+F_{\beta \alpha}^{\sigma}(\mathbf{k})\right) .
\end{gathered}
$$

Solving this set of equations, we obtain

$$
\begin{gathered}
F_{\alpha \alpha}^{\sigma}(\mathbf{k})=\frac{\omega-E_{0 \sigma}-A_{\bar{\sigma}}}{\left(\omega-E_{0 \sigma}\right)\left(\omega-E_{0 \sigma}-2 A_{\bar{\sigma}}\right)}, \\
F_{\beta \alpha}^{\sigma}(\mathbf{k})=F_{\alpha \alpha}^{\sigma}(\mathbf{k})-\frac{1}{\omega-E_{0 \sigma}} .
\end{gathered}
$$

The poles of $F_{\alpha \alpha}^{\sigma}(\mathbf{k})$ determine the spectrum $E_{ \pm}^{\sigma}(\mathbf{k})$, given by Eqs. (8) in the main text. In terms of $E_{ \pm}^{\sigma}(\mathbf{k})$, the Green's functions of $f$ electrons are

$$
\begin{aligned}
F_{\alpha \alpha}^{\sigma}(\mathbf{k})= & \frac{\omega-E_{0 \sigma}-A_{\bar{\sigma}}}{\left(\omega-E_{0 \sigma}\right)\left(\omega-E_{0 \sigma}-2 A_{\bar{\sigma}}\right)}=\frac{1}{2\left(\omega-E_{0 \sigma}\right)} \\
& -\frac{1}{2 W_{\sigma}(\mathbf{k})}\left[\frac{\varepsilon_{\mathbf{k} \sigma}-E_{+}^{\sigma}(\mathbf{k})}{\omega-E_{+}^{\sigma}(\mathbf{k})}-\frac{\varepsilon_{\mathbf{k} \sigma}-E_{-}^{\sigma}(\mathbf{k})}{\omega-E_{-}^{\sigma}(\mathbf{k})}\right] .
\end{aligned}
$$

and

$$
\begin{aligned}
F_{\beta \alpha}^{\sigma}(\mathbf{k})= & F_{\alpha \alpha}^{\sigma}(\mathbf{k})-\frac{1}{\omega-E_{0 \sigma}}=-\frac{1}{2\left(\omega-E_{0 \sigma}\right)} \\
& -\frac{1}{2 W_{\sigma}(\mathbf{k})}\left[\frac{\varepsilon_{\mathbf{k} \sigma}-E_{+}^{\sigma}(\mathbf{k})}{\omega-E_{+}^{\sigma}(\mathbf{k})}-\frac{\varepsilon_{\mathbf{k} \sigma}-E_{-}^{\sigma}(\mathbf{k})}{\omega-E_{-}^{\sigma}(\mathbf{k})}\right],
\end{aligned}
$$

where $W_{\sigma}(\mathbf{k}) \equiv E_{+}^{\sigma}(\mathbf{k})-E_{-}^{\sigma}(\mathbf{k})$.

We next calculate $G_{c \alpha}^{\sigma}(\mathbf{k})$. Using the corresponding equation of motion

$$
\left(\omega-\varepsilon_{\mathbf{k} \sigma}\right) G_{c \alpha}^{\sigma}(\mathbf{k})=\alpha_{\bar{\sigma}}\left(F_{\alpha \alpha}^{\sigma}(\mathbf{k})+F_{\beta \alpha}^{\sigma}(\mathbf{k})\right),
$$

we obtain

$$
\begin{aligned}
G_{c \alpha}^{\sigma}(\mathbf{k})= & \frac{\alpha_{\bar{\sigma}}}{\left(\omega-\varepsilon_{\mathbf{k} \sigma}\right)\left(\omega-E_{0 \sigma}-2 A_{\bar{\sigma}}\right)}=\frac{\alpha_{\bar{\sigma}}}{W_{\sigma}(\mathbf{k})}\left[\frac{1}{\omega-E_{+}^{\sigma}(\mathbf{k})}\right. \\
& \left.-\frac{1}{\omega-E_{-}^{\sigma}(\mathbf{k})}\right] .
\end{aligned}
$$

Finally, the Green's functions for itinerant electrons $G_{c c}^{\sigma}(\mathbf{k})$ are calculated in the same way, starting with

$$
\begin{gathered}
\left(\omega-\varepsilon_{\mathbf{k} \sigma}\right) G_{c c}^{\sigma}(\mathbf{k})=\alpha_{\bar{\sigma}}\left(G_{\alpha c}^{\sigma}(\mathbf{k})+G_{\beta c}^{\sigma}(\mathbf{k})\right)+1, \\
\left(\omega-E_{0 \sigma}\right) G_{\alpha c}^{\sigma}(\mathbf{k})=\alpha_{\bar{\sigma}} G_{c c}^{\sigma}(\mathbf{k}),
\end{gathered}
$$

and solving Eqs. (A8), we obtain

$$
G_{c c}^{\sigma}(\mathbf{k})=-\frac{1}{W_{\sigma}(\mathbf{k})}\left[\frac{E_{0 \sigma}-E_{+}^{\sigma}(\mathbf{k})}{\omega-E_{+}^{\sigma}(\mathbf{k})}-\frac{E_{0 \sigma}-E_{-}^{\sigma}(\mathbf{k})}{\omega-E_{-}^{\sigma}(\mathbf{k})}\right] .
$$

*Passed away on November 15, 2006 during the final stages of this work.

${ }^{1}$ H. Tsunetsugu, M. Sigrist, and K. Ueda, Rev. Mod. Phys. 69, 809 (1997).

${ }^{2}$ G. R. Stewart, Rev. Mod. Phys. 56, 755 (1984).

${ }^{3}$ B. Coqblin, C. Lacroix, M. S. Gusmão and J. R. Iglesias, Phys. Rev. B 67, 064417 (2003).

${ }^{4}$ B. Coqblin, M. D. Núñez-Regueiro, A. Theumann, J. R. Iglesias, and S. G. Magalhaes, Philos. Mag. 86, 2567 (2006).

${ }^{5}$ P. Coleman and N. Andrei, J. Phys.: Condens. Matter 1, 4057 (1989).

${ }^{6}$ J. R. Iglesias, C. Lacroix, and B. Coqblin, Phys. Rev. B 56, 11820 (1997)
${ }^{7}$ S. Doniach, Proceedings of the International Conference on Valence Instabilities and Related Narrow-Band Phenomena, edited by R. D. Parks (Plenum, New York, 1976), p. 168.

${ }^{8}$ J. Rossat-Mignod, L. P. Regnault, J. L. Jacoud, C. Vettier, P. Lejay, J. Flouquet, E. Walker, D. Jaccard, and A. Amato, J. Magn. Magn. Mater. 76-77, 376 (1988).

${ }^{9}$ J. Schoenes, J. Less-Common Met. 121, 87 (1986).

${ }^{10}$ J. Schoenes, B. Frick, and O. Vogt, Phys. Rev. B 30, 6578 (1984).

${ }^{11}$ Z. Bukowski, R. Troc, J. Stepien-Damm, C. Sulkowski, and V. H. Tran, J. Alloys Compd. 403, 65 (2005).

${ }^{12}$ V. H. Tran, R. Troc, Z. Bukowski, D. Badurski and C. Sulkowski, Phys. Rev. B 71, 094428 (2005).

${ }^{13}$ V. H. Tran, S. Paschen, F. Steglich, R. Troc, and Z. Bukowski, 
Phys. Status Solidi B 243, 94 (2006).

${ }^{14}$ D. Aoki, A. Huxley, E. Ressouche, D. Braithwaite, J. Flouquet, J. P. Brison, E. Lhotel, and C. Paulsen, Nature (London) 413, 613 (2001).

${ }^{15}$ J. Flouquet, Prog. Low Temp. Phys. 15, 139 (2006).

${ }^{16}$ W. H. Lee, H. C. Ku, and R. N. Shelton, Phys. Rev. B 38, 11562 (1988).

${ }^{17}$ A. Eiling and J. S. Schilling, Phys. Rev. Lett. 46, 364 (1981).

${ }^{18}$ Nevertheless, we should notice that a kind of coexistence of ferromagnetism and Kondo-like behavior has been observed too in a few Ce-based dense Kondo systems, such as $\mathrm{CePt}_{x} \mathrm{Si}$ compound (Ref. 16) or CeAg one (Ref. 17). But the Curie temperatures of these compounds are relatively small, typically of order $5 \mathrm{~K}$, and this result can be considered as a clear sign of a strong competition rather than a real coexistence between the Kondo effect and ferromagnetic order.

${ }^{19}$ J. Schoenes, O. Vogt, J. Lohle, F. Hulliger, and K. Mattenberger, Phys. Rev. B 53, 14987 (1996).

${ }^{20}$ J. Schoenes, M. Broschwitz, K. Litfin, S. Heathman, O. Vogt, and K. Mattenberger (private communication).

${ }^{21}$ Q. G. Sheng and Bernard R. Cooper, J. Magn. Magn. Mater. 164, 335 (1996).

${ }^{22}$ T. Durakiewicz et al., Phys. Rev. Lett. 93, 267205 (2004).

${ }^{23}$ G. Zwicknagl, A. N. Yaresko, and P. Fulde, Phys. Rev. B 65, 081103(R) (2002).

${ }^{24}$ G. Zwicknagl, A. N. Yaresko, and P. Fulde, Phys. Rev. B 68, 052508 (2003).

${ }^{25}$ L. V. Pourovskii, M. I. Katsnelson, and A. I. Lichtenstein, Phys. Rev. B 72, 115106 (2005).

${ }^{26}$ J. Gan, P. Coleman, and N. Andrei, Phys. Rev. Lett. 68, 3476 (1992).

${ }^{27}$ P. D. Sacramento and P. Schlottmann, Phys. Rev. B 40, 431 (1989).

${ }^{28}$ P. Schlottmann and P. D. Sacramento, Adv. Phys. 42, 641 (1993).

${ }^{29}$ N. B. Perkins, M. D. Núñez-Regueiro, J. R. Iglesias, and B. Co- qblin, Physica B 378-380, 698 (2006).

${ }^{30}$ N. Andrei and E. Orignac, Phys. Rev. B 62, R763 (2000).

${ }^{31}$ S. Florens, Phys. Rev. B 70, 165112 (2004).

${ }^{32}$ S. Di Matteo, N. B. Perkins, and C. R. Natoli, Phys. Rev. B 65, 054413 (2002).

${ }^{33}$ G. Honner and M. Gulacsi, Z. Phys. B: Condens. Matter 104, 733 (1997).

${ }^{34} \mathrm{~A}$ ferromagnetic coupling between localized magnetic moments may result from two contributions: an effective RKKY interaction and, possibly, a direct exchange. The RKKY interaction may lead to ferromagnetic ordering for strong coupling regime, large $\left|J_{K}\right|$, and only for partial filling of the conduction band, as it has been shown by many authors for the usual KL model, see, for example, Refs. 1 and 33.

${ }^{35}$ S. Burdin, A. Georges, and D. R. Grempel, Phys. Rev. Lett. 85, 1048 (2000).

${ }^{36}$ S. Burdin, thesis, Université Joseph Fourier, Grenoble, 2001.

${ }^{37}$ P. Nozières, Eur. Phys. J. B 6, 447 (1998).

${ }^{38}$ V. Barzykin, Phys. Rev. B 73, 094455 (2006).

${ }^{39}$ C. Lacroix and M. Cyrot, Phys. Rev. B 20, 1969 (1979).

${ }^{40}$ D. Kaczorowski, Solid State Commun. 99, 949 (1998).

${ }^{41}$ A. Das, S. K. Paranjpe, P. Raj, A. Satyamoorthy, K. Shashikala, and S. K. Malik, Solid State Commun. 114, 87 (2000).

${ }^{42}$ T. Taniguchi, H. Morimoto, Y. Miyako, and S. Ramakrishnan, J. Magn. Magn. Mater. 177-181, 55 (1998).

${ }^{43}$ D. Kaczorowski (private communication).

${ }^{44}$ Y. Dalichaouch, M. B. Maple, M. S. Torikachvili, and A. L. Giorgi, Phys. Rev. B 39, 2423 (1989).

${ }^{45}$ M. S. Torikachvili, L. Rebelsky, K. Motoya, S. M. Shapiro, Y. Dalichaouch, and M. B. Maple, Phys. Rev. B 45, 2262 (1992).

${ }^{46}$ E. D. Bauer, V. S. Zapf, P. C. Ho, N. P. Butch, E. J. Freeman, C. Sirvent, and M. B. Maple, Phys. Rev. Lett. 94, 046401 (2005).

${ }^{47}$ E. Colineau, F. Wastin, J. P. Sanchez, and J. Rebizant (private communication). 